\title{
Editorial
}

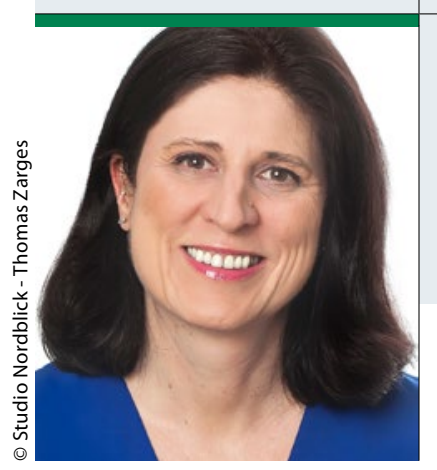

„Unsere Patienten benötigen Unterstützung und Beratung

auf ihrem Weg durch den Dschungel aus manchmal

hilfreichen, manchmal aber auch verwirrenden Informationen."

PD Dr. med. Georgia Schilling

Abteilung Onkologie mit Sektion Hämatologie, Asklepios Klinik Altona,

Hamburg

\section{Umgang mit der Informationsflut}

$\mathrm{M}$ it der Diagnose Krebs beginnt für Patienten und Angehörige die Suche nach der bestmöglichen Therapie und dem geeigneten Spezialisten. Doch im Zeitalter moderner Medien fällt es schwer, aus der Fülle an Beiträgen zum Thema Krebs seriöse und relevante Informationen herauszufiltern. Patienten wollen vermehrt in Therapieentscheidungen einbezogen werden. Hierfür benötigen sie entsprechende Informationen - z. B. über Krankheiten und deren Ursachen, über Therapien und deren Nebenwirkungen. Gleichzeitig setzt auch die Gesundheitspolitik auf den ,informierten Patienten“: Dem Urteil einschlägiger Experten zufolge wirken sich umfassendere und bessere Informationen positiv auf den Therapieerfolg aus und ermöglichen zudem Einsparungen im Gesundheitswesen.

\section{Über die Hälfte der Deutschen ist mit der Informationsflut überfordert!}

Überfordert sind in erster Linie ältere Menschen, Menschen mit Migrationshintergrund oder niedrigerem Bildungsniveau. Ca. $44 \%$ weisen einer aktuellen Studie zufolge eine unzureichende und weitere $10 \%$ eine eingeschränkte Gesundheitskompetenz auf [https://tinyurl.com/y957gymb]. Damit liegt Deutschland klar unter dem europäischen Durchschnitt.

Die Folgen einer geringen Gesundheitskompetenz sind:

_ein höheres Risiko ins Krankenhaus zu kommen und länger dort zu bleiben;

_eine geringere Compliance;

_eine höhere Fehlerrate bei der Einnahme von Medikamenten;

_ mehr und stärkere Krankheitssymptome bei der

Erstvorstellung beim Arzt;

_ein geringerer Behandlungserfolg.

\section{Hilft Dr. Google?}

Die Patienten beklagen zu geringe Information über Behandlungsoptionen und alternative Behandlungsmöglichkeiten, Nebenwirkungen und Spätfolgen von
Therapien. Auch darüber, was sie selbst für sich tun können - im Sinne von (tertiär-)präventiven Maßnahmen -, um ihr Rezidivrisiko oder das Auftreten eines Zweittumors zu verhindern, möchten sie besser aufgeklärt werden. „Dr. Google“ scheint hier der ideale Berater zu sein, ist er doch 24 Stunden am Tag, 7 Tage die Woche und das an 365 Tagen im Jahr niederschwellig verfügbar ... Der schnelle Zugang zu immer mehr Informationen im Internet ist dabei Chance und Herausforderung zugleich, denn im Internet kann der Laie neueste wissenschaftliche Forschungsergebnisse nicht immer von gewerblichen Angeboten und interessengeleiteten Empfehlungen unterscheiden. Patientenforen und Chats sind mit individuellen, häufig negativen Erfahrungen belastet und können so Ängste, aber auch ganz falsche Erwartungen an das Behandlungsteam auslösen.

Unsere Patienten benötigen also Unterstützung und Beratung auf ihrem Weg durch den Dschungel aus manchmal hilfreichen, manchmal aber auch verwirrenden oder fehlleitenden Informationen.

Das direkte Gespräch mit dem Patienten und seinen Angehörigen ist daher auch im Zeitalter moderner Medien immer noch der beste Weg, den Unterstützungsbedarf herauszufinden und relevante Informationen weiterzugeben. Wir müssen uns auf ärztlicher Seite darauf einstellen, dass Patienten in Zukunft mit einer höheren Erwartungshaltung und mehr (Fehl-)Informationen zu uns kommen. Daher ist es an uns, das Angebot medizinischer Information patientenfreundlicher zu gestalten und uns auf die unterschiedlichen Ansprüche und Fähigkeiten der verschiedenen Patientengruppen einzustellen.

Patienten brauchen Zugang zu qualitätsgesicherten Informationen und müssen darüber hinaus geschult und dabei unterstützt werden, sie zu beurteilen. Dies ist nur durch eine persönliche Beratung möglich. Nur dann werden die Schlagworte Patientenorientierung und Patientenbeteiligung Realität in der Versorgung!

Georgia Schilling 